\title{
Study of the Dispersion of Platinum Nanoparticles in Nanoporous Carbon
}

\author{
Ramakrishnan Rajagopalan ${ }^{1}$, Henry C. Foley ${ }^{1}$ and Lawrence F. Allard ${ }^{2}$ \\ ${ }^{1}$ Department of Chemical Engineering, Pennsylvania State University, PA, 16801 \\ ${ }^{2}$ Oakridge National Laboratory, TN, 37831
}

Nanoporous carbon (NPC) possess enormous potential in the area of catalysis and gas separations due to their unique size and shape selectivity. The ease of formation and their high temperature stability facilitates the use of these carbons as templates for supporting catalytic materials such as Platinum. The ability to form supported catalytic nanoporous membranes with this material expands the horizon of engineering applications that is not limited to gas separation and catalysis. One of the promising applications includes the use of platinum supported nanoporous carbon (Pt/NPC) membranes as electrodes in proton exchange membrane fuel cells (PEM). These materials present graphite-like domains that grant the necessary electrical conductivity along with the porous network, which allows the access of the gases to the catalytically active particles of platinum. Pt/NPC was prepared by pyrolyzing the suspension of platinum precursor dispersed in polyfurfuryl alcohol under inert atmosphere.

Preliminary investigations of the platinum loaded nanoporous carbon indicate that the size of the platinum particles embedded in the carbon ranges from 4-5 $\mathrm{nm}$. It was interesting to note that the size of the platinum particles was independent of the platinum loading. This was also in agreement with the particle size calculated using X-ray diffraction patterns. The platinum particles had a tendency to form clusters. The size of the platinum clusters increased with platinum loading. The presence of platinum particles in the carbon also led to the local rearrangement of the structure of carbon. There was evidence for local graphitization of carbon around the platinum nanoparticles. This could be attributed to the templating effect of the platinum particles in the carbon matrix. 


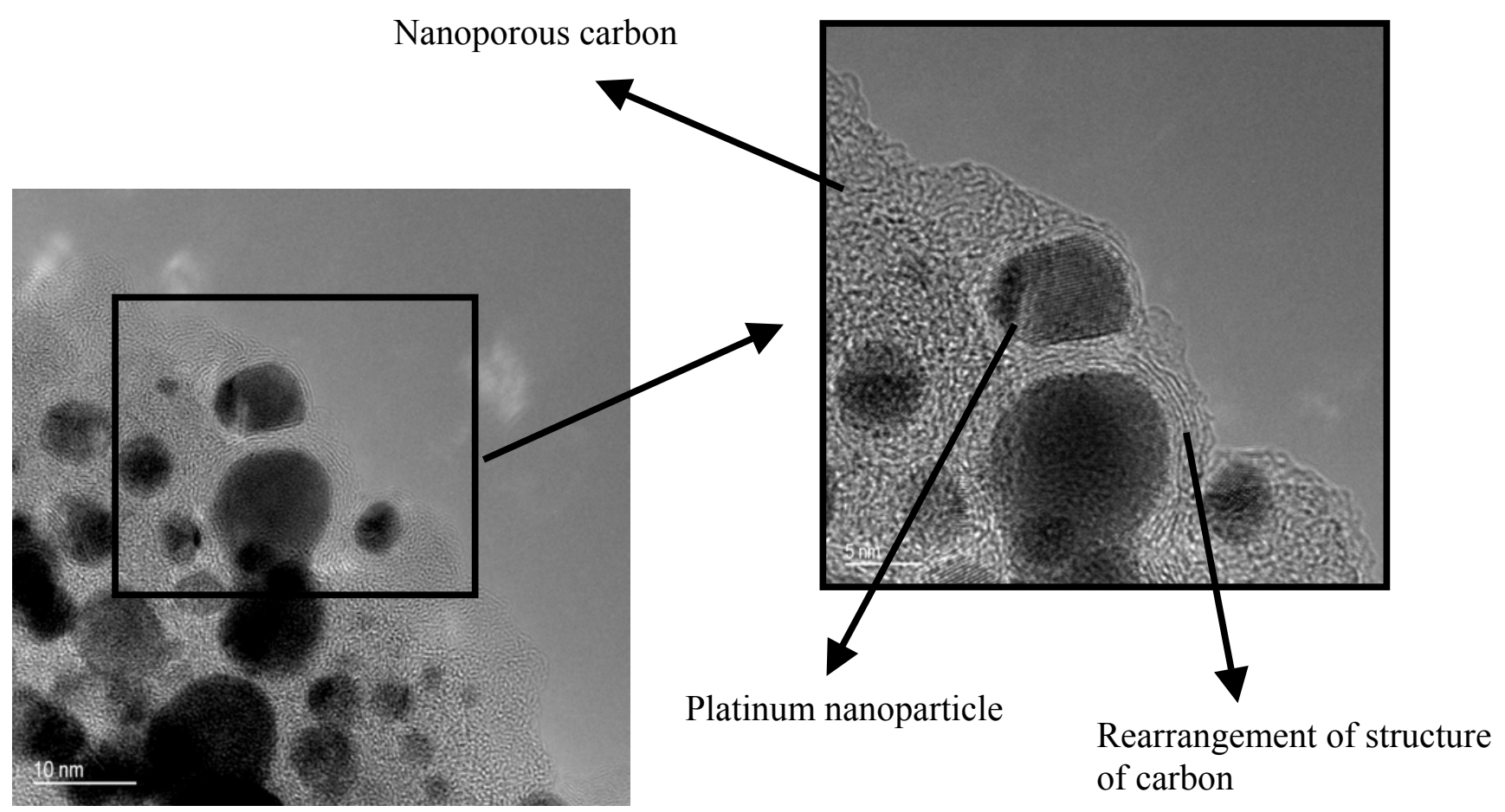

Figure 1. HRTEM image of $0.675 \mathrm{wt} \%$ Platinum loaded nanoporous carbon

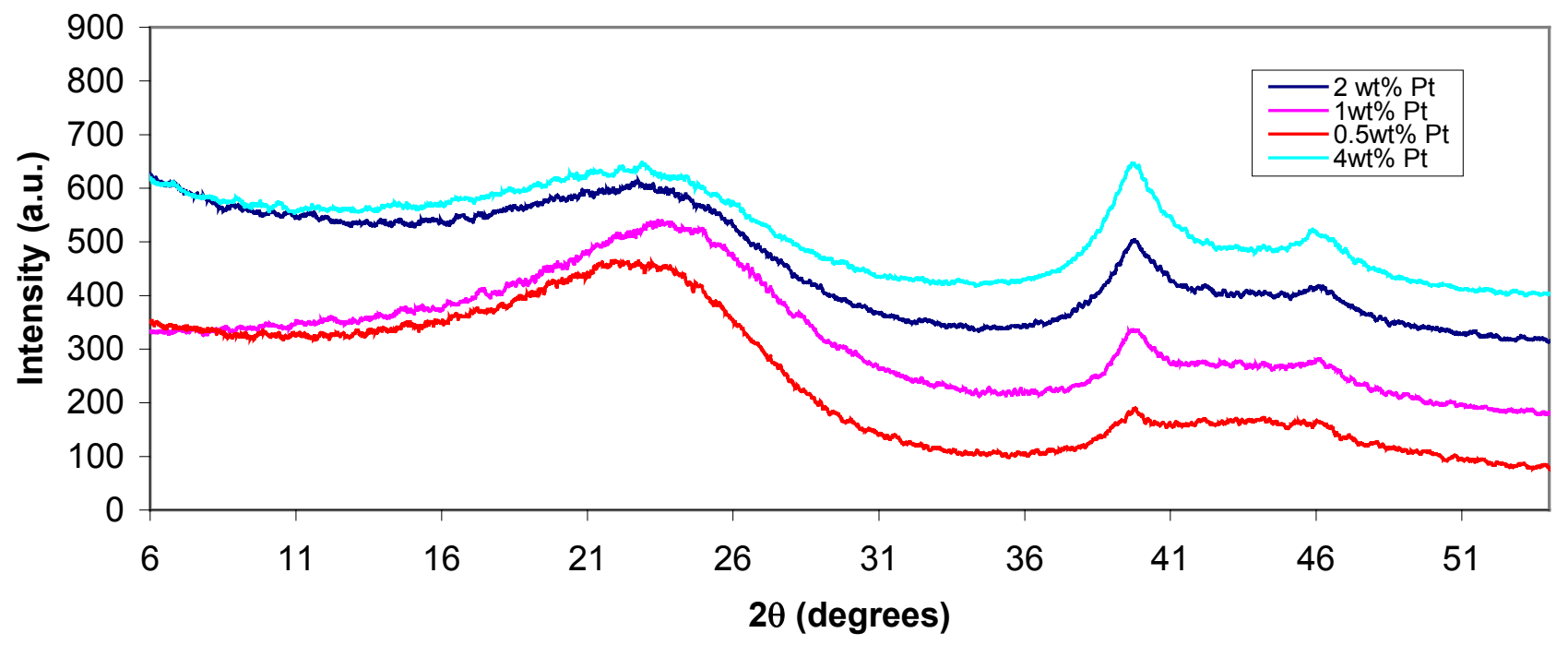

Figure 2. X-ray Diffraction pattern of platinum loaded nanoporous carbon as a function of platinum loading 